\title{
Effect of Transplantation Site on the Results of Pancreatic Islet Isografts in Diabetic Rats
}

\author{
C.B. Kemp, M.J. Knight, D.W. Scharp, W.F. Ballinger and P.E. Lacy \\ Departments of Surgery and Pathology, Washington University School of Medicine, St. Louis, Missouri, USA \\ Received: May 24, 1973, and in revised form: July 14, 1973
}

\begin{abstract}
Summary. Isolated pancreatic islets were transplanted isogeneically into the subcutaneus tissue, peritoneum and portal vein of diabetic rats. Implantation of islets subcutaneously did not modify the diabetic state. Intraperitoneal islets ameliorated the effects of diabetes but normal urine volumes, blood glucose and urine glucose levels were not achieved. Direct injection of islets into the portal vein resulted in normal urine volumes, normo-
\end{abstract}

glycemia and abolition of glycosuria in the rats studied. These effects have been maintained 2 months later. - It is suggested that the portal environment may be the most effective site for transplanted pancreatic islets.

Key words: Transplantation, islets of Langerhans, diabetes mellitus, portal vein, pancreas, isograft
Although insulin maintenance therapy has prolonged the life of the diabetic and significantly reduced deaths from ketoacidosis, the disabling late complications of the disease are not prevented by this form of treatment. Furthermore, in some patients, especially in the juvenile diabetic group, it is often difficult to maintain blood sugar levels within the normal range with exogenous insulin, indicating the need for an alternative method of treatment.

Recently there has been much interest in the possibility of pancreatic islet transplants in the treatment of diabetes, and experimental work by Ballinger and Lacy [1] suggests that this approach is now feasible. These workers showed that intraperitoneal and intramuscular implantation of isolated pancreatic islets ameliorates the effects of streptozotocin induced diabetes in the rat. However, although the intraperitoneal and intramuscular siting of these islets reduced hyperglycaemia, polyuria and glycosuria in these animals, consistently normal values were rarely achieved.

Under normal circumstances insulin from the pancreatic cells is secreted directly into the portal venous system. We have investigated the possibility that this intraportal site may provide a more physiological environment for the transplanted islets and possibly more effective utilization of secreted hormones than can be achieved by siting the islets intraperitoneally or subcutaneously.

\section{Materials and Methods}

\section{Donors}

Pancreatic islets were harvested from inbred Lewis rats $(250-300 \mathrm{~g})$ by the collagenase digestion method of Lacy and Kostianorsky [2]. This method consists of cannulation of the common bile duct, distension of the pancreas with a balanced salt solu- tion, fine chopping of the excised gland, and digestion for approximately ten minutes at $37^{\circ} \mathrm{C}$ with collagenase in a shaker water bath. After a series of washes, the islets were separated from unwanted acinar tissue by differential layering of dialyzed Ficoll [4]. This technique requires dialyzing Ficoll, a high molecular weight polymer of sucrose, against distilled water to remove various contaminants with lyophilization back to a powder. The washed digest preparation is mixed with dialyzed Ficoll $25 \%$ (W.V.). Careful layering of decreasing concentrations of dialyzed Ficoll $(23 \%, 20 \%$ and $11 \%$ ) above the preparation readies it for centrifugation for $10 \mathrm{~min}$ at $800 \mathrm{~g}$. The islets rise through the density gradient to settle at the $20 \%-11 \%$ interface leaving the digested acinar tissue behind in the $25 \%$ layer. A clean separation of islets is rapidly achieved with this technique. The number of islets for each preparation is estimated after centrifugation by the appearance of the density of islets at the $11 \%$ $20 \%$ interface. Frequent checking confirms the estimate to be accurate within 50 islets for each isolation. The islets can then be easily removed from the Ficoll with a siliconized transfer pipet and readied for transplantation.

\section{Recipients}

Twenty-five previously healthy Lewis rats (250$300 \mathrm{~g}$ ) of the same inbred strain as the donor group were made diabetic by the intravenous injection of streptozotocin (Upjohn) at a dose schedule of $65 \mathrm{mg} / \mathrm{kg}$. These rats returned to their metabolic cages for 3 weeks during which time a free diet of rat chow and water was allowed. Urine volumes were measured daily; blood sugars and urine glucose concentrations were estimated twice weekly. After 3 weeks each of the 25 rats was allotted randomly to one of 4 groups.

Group I. Diabetic Controls (10 rats)

These rats received no treatment for diabetes. 
Group II. Subcutaneous Implantation (5 rats)

Under ether anaesthesia a No. 23 needle was introduced through the skin of the anterior abdominal wall and between $850-970$ (Mean 890) islets deposited subcutaneously.

Group III. Intraperitoneal Implantation (5 rats)

Rats were anaesthetized with ether. Through a small $(1 \mathrm{~cm})$ midline abdominal incision between 600 and 850 (Mean 770) islets were introduced into the peritoneal cavity and the wound closed.

Group IV. Intraportal Implantation (5 rats)

A long $(3 \mathrm{~cm})$ midline abdominal incision was cages for a period of 3 months during which time a free diet of rat chow and water was allowed. Urine volumes and glucose concentrations were measured daily and blood sugar levels were estimated twice weekly.

$$
\text { Results (Figs. 1-3) }
$$

Group I. All 10 control rats were grossly diabetic within $24 \mathrm{~h}$ of streptozotocin administration and remained so for the period of study. There was no improvement of this diabetic state with the passage of time. Blood sugar levels fluctuated between 280 and

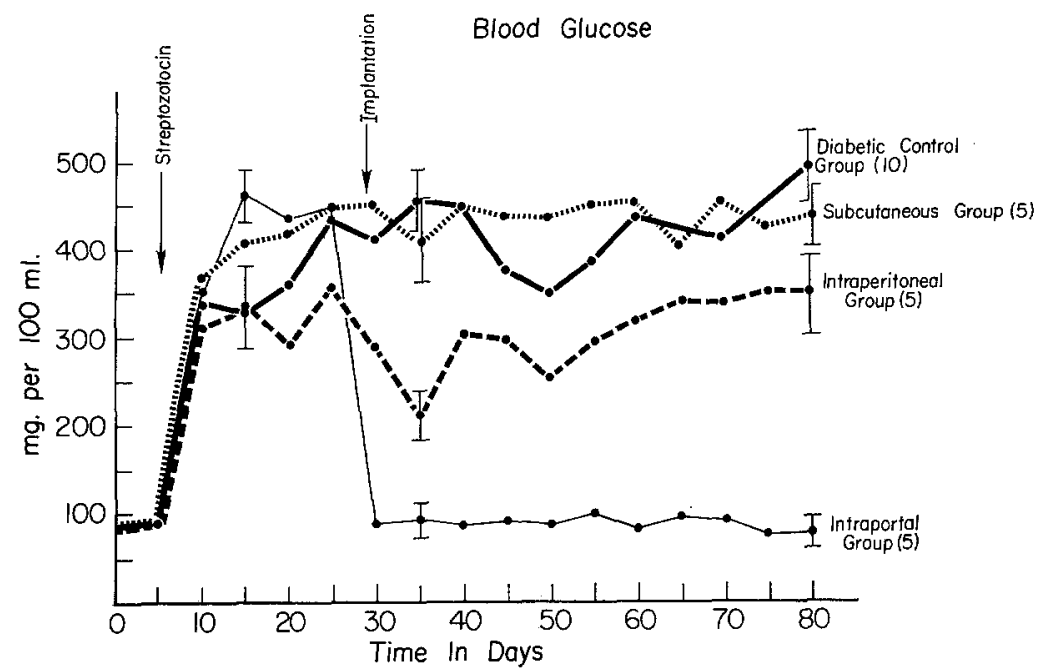

Fig. 1. Effect of portal vein [5], subcutaneous [5] and intraperitoneal [5] implantation of pancreatic islets on the blood glucose of diabetic rats. Diabetic controls [10]

made under ether anaesthesia. Displacement of the large and small bowel to the left side displayed the portal and superior mesenteric veins with ease. A siliconized $1 \mathrm{ml}$ glass syringe with attached No. 23 needle was rinsed with Hanks solution and the plunger withdrawn to the $0.75 \mathrm{ml}$ marking on the empty syringe barrel. The curved needle was then introduced cephalad into the portal vein for a distance of $1 \mathrm{~cm}$ and the plunger removed from the syringe barrel. $400-600$ (Mean 550) islets in suspension were then introduced into the $0.3 \mathrm{ml}$ of blood which had entered the syringe barrel, and the plunger replaced. With gentle pressure the blood and contained islets were injected into the portal vein, care being taken to avoid the introduction of air. Blood was then withdrawn a second time into the syringe and reinjected to deal with any residual islets. The needle was removed and pressure applied over the venipuncture for about 3 min by which time bleeding had usually ceased. After replacing the bowel the abdomen was closed.

Rats from all groups were observed in metabolic
$700 \mathrm{mg}$ per $100 \mathrm{ml}$ with daily urine volumes ranging from 62 to $168 \mathrm{ml}$. Heavy glycosuria $(4-16.6 \mathrm{~g}$ per day) reflected the hyperglycaemia.

Group II. Subcutaneous implantation of islets did not alter the diabetic state of the 5 rats in this group Urine volumes remained high $(70-160 \mathrm{ml}$ per day with blood sugar levels fluctuating between 300 and $710 \mathrm{mg}$ per $100 \mathrm{ml}$. Urine glucose was unchanged at $4-15 \mathrm{~g}$ per day.

Group III. Intraperitoneal implantation of pancreatic islets had an ameliorating effect on the diabetic state in all 5 rats. Blood glucose levels fell to $200-$ $400 \mathrm{mg}$ per $100 \mathrm{ml}$ and a similar reduction in urine volumes was also noted $(70-100 \mathrm{ml}$ per day). Urinary glucose content was reduced to $1.8-6.8 \mathrm{~g}$ per day.

Group IV. Injection of islets directly into the portal vein resulted in normoglycaemia $(62-121 \mathrm{mg}$ per $100 \mathrm{ml}$ ) normal urine volume $(6-21 \mathrm{ml}$ per day) and abolition of glycosuria in the 5 rats studied. This condition is maintained 2 months later. 


\section{Discussion}

Previous work has shown that pancreatic islets isolated by the collagenase digestion method maintain their insulin secreting properties in vitro [3] and that intraperitoneal transplantation of such islets reduces the severity of experimental diabetes in the rat [1]. islets into a subcutaneous pocket had no effect on the diabetic state of recipient rats.

These results suggest that the site of implantation may be an important consideration if transplantation of pancreatic islets is to be used in the treatment of clinical diabetes. The ideal implantation site would be one which would permit maximum survival and

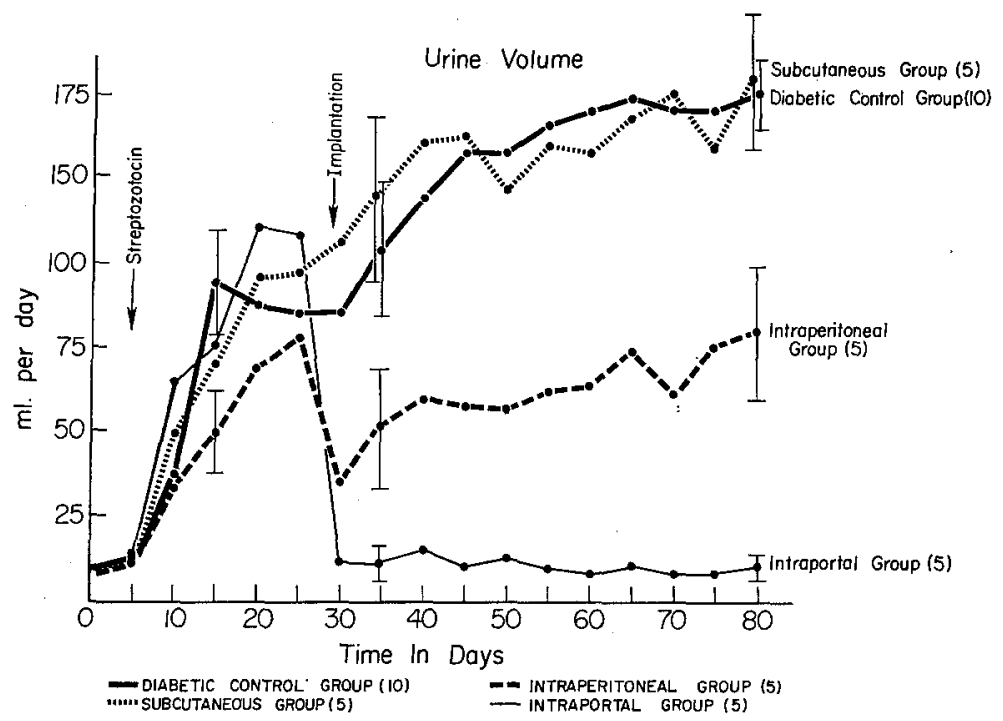

Fig. 2. Effect of portal vein [5], subcutaneous [5] and intraperitoneal [5] implantation of pancreatic islets on the urine volume of diabetic rats. Diabetic controls [10]

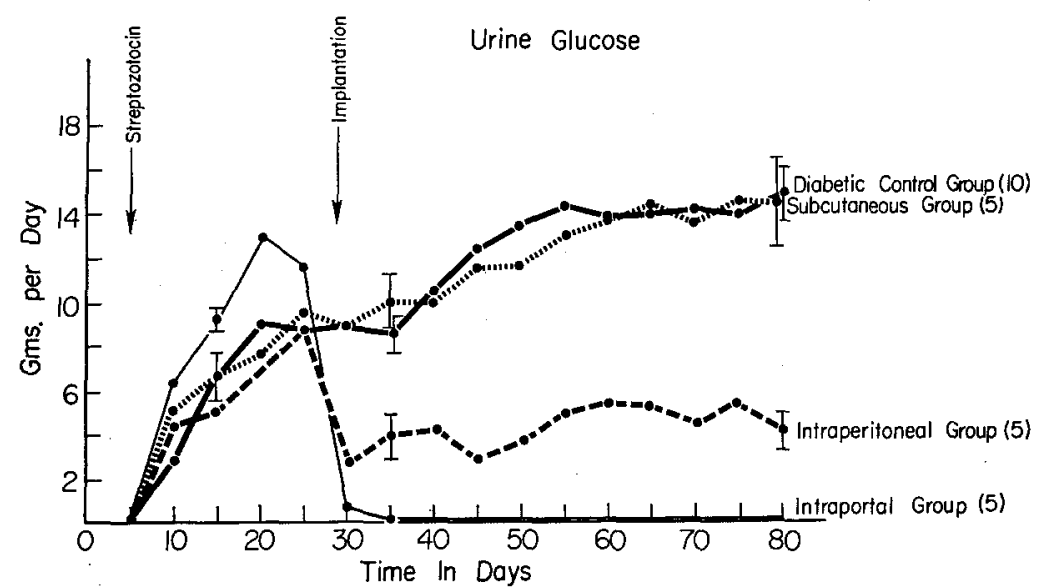

Fig. 3. Effect of portal vein [5], subcutaneous [5] and intraperitoneal [5] implantation of pancreatic islets on the urine glucose of diabetic rats. Diabetic controls [10]

This ameliorating effect of intraperitoneal isografts is confirmed by the present study although normal values for blood sugar, urine volume and urine glucose were not achieved. Implantation of the same number of islets into the portal venous system, however, resulted in normoglycaemia, normal urine volumes and abolition of glycosuria, effects which have been maintained two months after transplantation. In contrast to this dramatic response, implantation of function of transplanted islets, maximum effectiveness of secreted hormones and safety to the patient. It would also be desirable that the transplanted islets be easily retrievable, particularly in view of the high infection risk associated with immunosupression therapy in diabetes. Perhaps the least hazardous implantation site in the diabetic patient would be a subcutaneous pocket; but the results of this study would indicate that islet function in this milieu would 


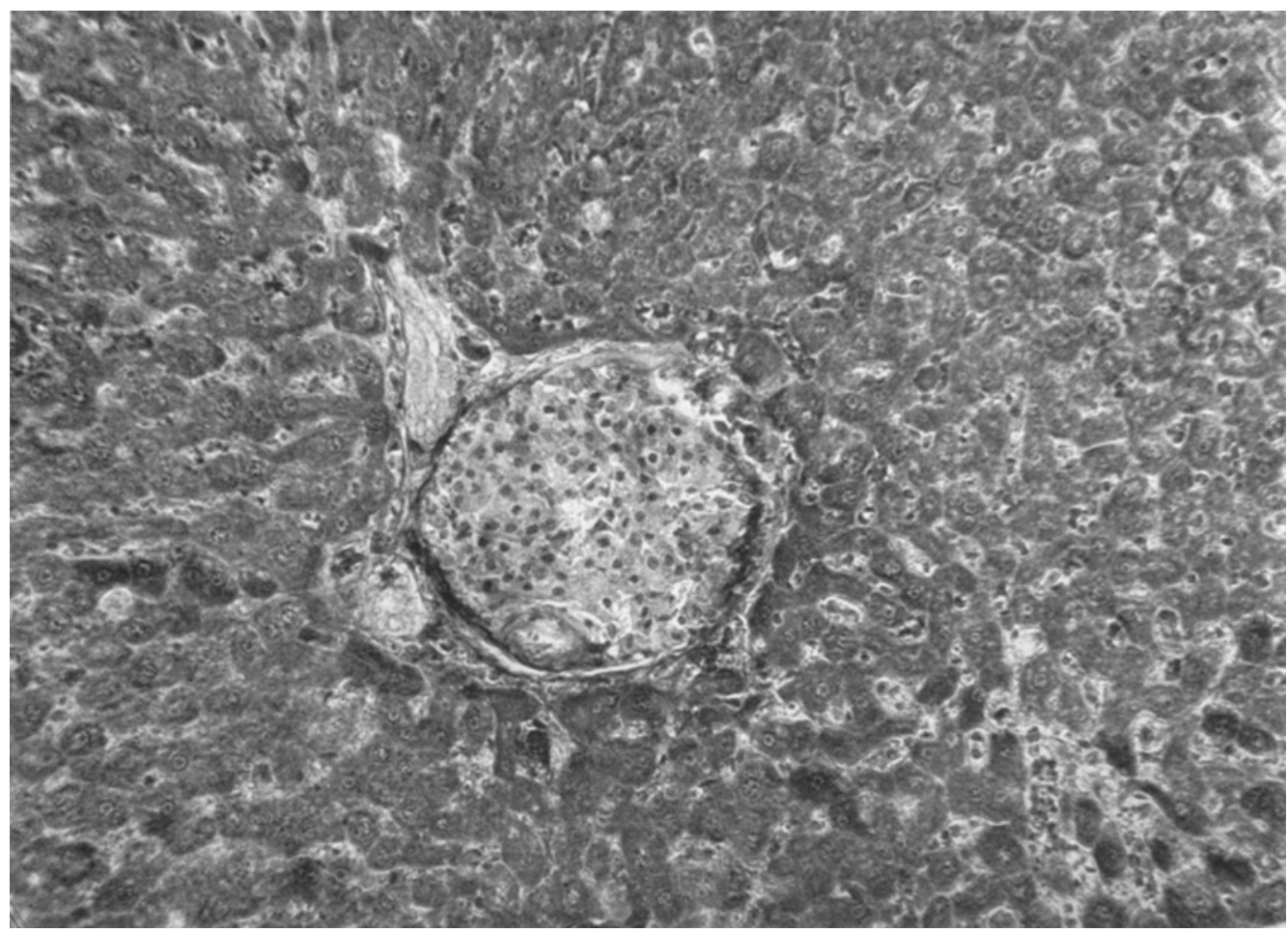

Fig. 4. Section of the liver showing a pancreatic islet in the terminal portal venule $24 \mathrm{~h}$ after injection into the portal vein. Aldehyde fuchsin $\times \mathbf{3 0 0}$

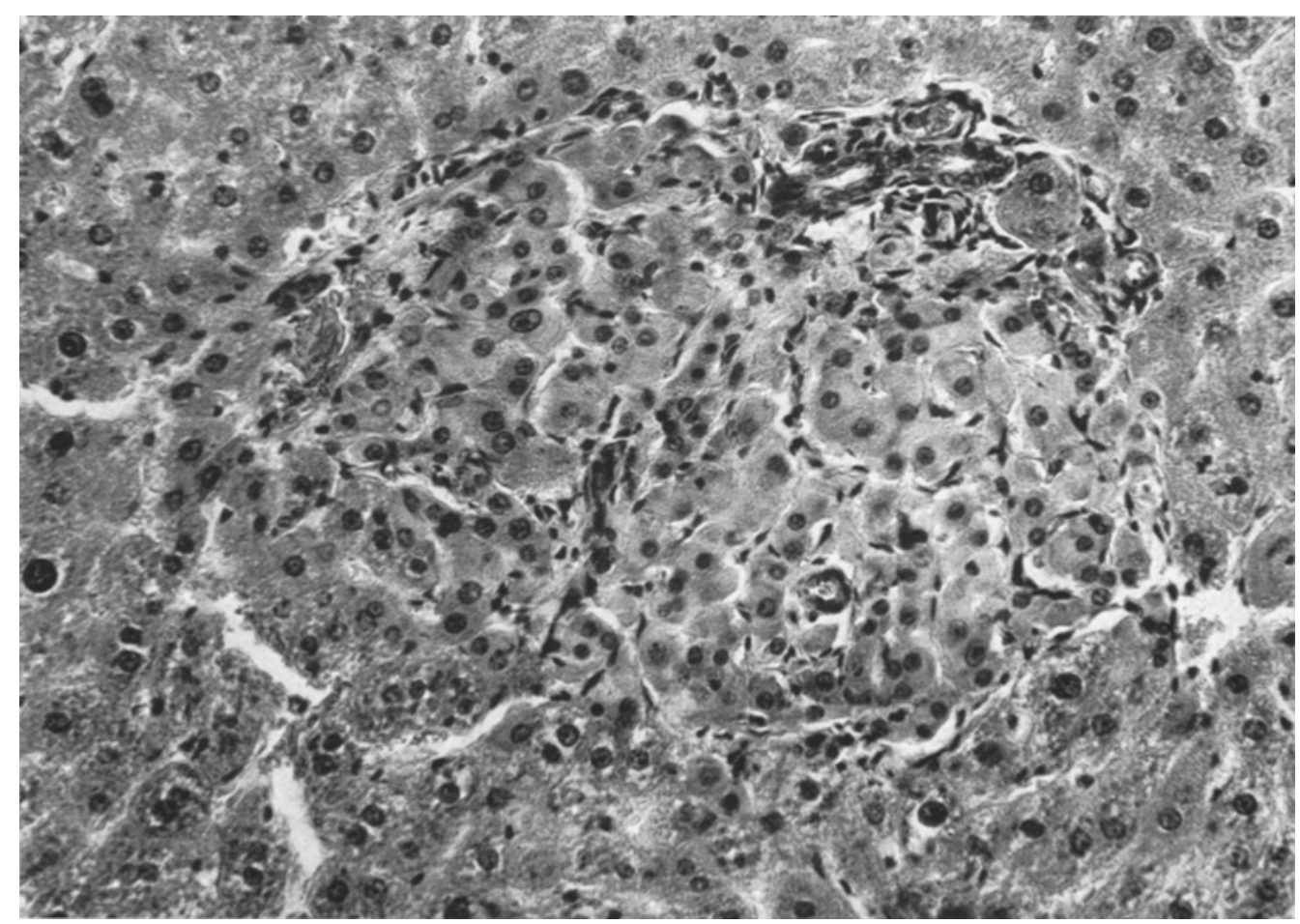

Fig. 5. Section of the liver 10 weeks following intraportal injection of pancreatic islets showing an islet in the connective tissue within a portal tract. Hematoxylin - eosin $\times 550$ 
be insufficient to materially affect the course of the diabetic state, possibly due to poor perfusion of the transplanted tissue. Similarly, the function of intraperitoneal islets would seem from this experimental study to be insufficient to maintain adequate control of blood sugar. Also, dissemination of tissue throughout the peritoneal cavity would present problems if retrieval were found to be necessary. Indeed, one is unable to locate the islets after intraperitoneal injection. The effect of siting the islets within the portal vascular system was dramatic with virtual recovery from the diabetic state in those rats studied. Whether regarding insulin release and hepatic utilization are in progress.

The dissemination of the transplanted islets throughout the liver may present problems when one considers this site for transplantation in man. It would seem desirable then if the intraportal environment is found to provide the most effective functional milieu, to design a system whereby transplanted islets are bathed in portal venous blood while at the same time being readily retrievable. Possibilities include selective portal vein injection in a larger organism such as man or the use of an intravascular

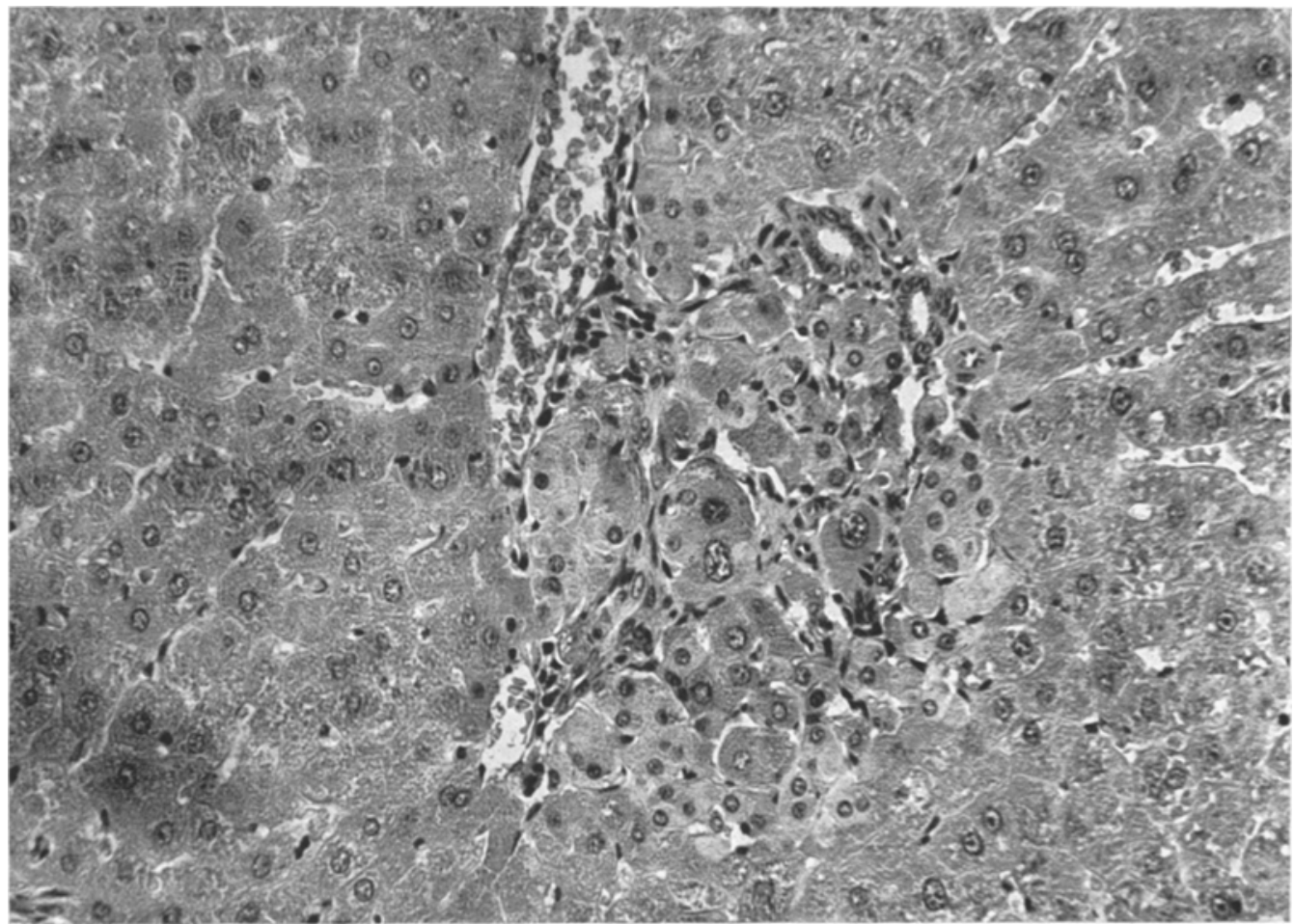

Fig. 6. Section of the liver 18 weeks after transplantation demonstrating normal beta cell granulation of an islet within the connective tissue of a portal tract. Aldehyde fuchsin $\times 400$

or not a similar response could have been achieved by injection of islets into the systemic circulation is as yet uncertain but preliminary experiments in this laboratory suggest that the intraportal environment is the more effective.

Investigations are underway to elucidate the sequence of events that allow for the establishment of the islets within the portal tract. Preliminary findings suggest that initially the islets are located in the terminal portal vessels $24 \mathrm{~h}$ after injection (Fig. 4). They become vascularized at some point while remaining within the portal tract (Fig. 5) and appear able to synthesize insulin as evidenced by beta cell granulation (Fig. 6). Their ability to satisfy the insulin needs of the recipient animal has been demonstrated by the clinical parameters presented. Detailed studies
Millipore chamber. However, to date there have been no reports of successful long term endocrine transplantation using the Millipore chamber. Alternatively, islets might be injected into the splenic artery which would allow tissue retrieval by splenectomy. Further investigations of these problems are at present in progress in this laboratory.

Acknowledgements. This study was supported by a grant from the Kroc Foundation.

Dr. Kemp is supported by a grant from the Cape Provincial Administration and Tygerberg Hospital, South Africa.

Dr. Knight is supported by a grant from St. George's Hospital, London and the Wellcome Foundation.

We gratefully acknowledge the technical assistance of Mrs. S.B. Nunnelley, Mr. R.W. Ratzlaff, Mrs. M.M. Feldmeier, Miss D.J. White, Mrs. R. Braitberg, Mr. G. J. Reed and Miss R. L. Langfeld. 


\section{References}

1. Ballinger, W.F., Lacy, P.F.: Transplantation of intact pancreatic islets in rats. Surgery $\mathbf{7 2}, \mathbf{1 7 5}-\mathbf{1 8 6}$ (1972)

2. Lacy, P.E., Kostianovsky, M.: A method for the isolation of intact islets of Langerhans from the rat pancreas. Diabetes 16, 35-39 (1967)

3. Lacy, P.E., Walker, M.M., Fink, C.J.: Perifusion of isolated rat islets in vitro. Diabetes $21,987-998(1972)$
4. Scharp, D.W., Kemp, C.B., Knight, M.J., Ballinger, W.F., Lacy, P.E.: The use of Ficoll in the preparation of viable islets of Langerhans from the rat pancreas. Submitted to Transplantation

Dr. C.B. Kemp

Department of Surgery

Tygerberg Hospital

Tiervlei Cape

South Africa 